\title{
Molecular differences in the $K R A S$ gene mutation between a primary tumor and related metastatic sites - case report and a literature review
}

\author{
Ewa Anna Kosakowska ${ }^{1}$, Rafał Stec ${ }^{2}$, Radosław Charkiewicz ${ }^{3}$, Marta Skoczek ${ }^{4}$, \\ Lech Chyczewski ${ }^{5}$
}

${ }^{1}$ Department of Gastrointestinal Cancer, Cancer Center-Institute, Warsaw, Poland

${ }^{2}$ Departament of Oncology, Military Institute of Medicine, Warsaw, Poland

${ }^{3}$ Department of Clinical Molecular Biology, Medical University of Bialystok, Bialystok, Poland

${ }^{4}$ Department of Medical Oncology, Cancer Center, Cracow, Poland

${ }^{5}$ Department of Clinical Pathology, Medical University of Bialystok, Bialystok, Poland

\begin{abstract}
In recent years the the set of diagnostic tools in colorectal cancers has been extended by the assessment of the KRAS gene status. Currently it is a necessary step in order to qualify patients for the targeted therapy. The results of the analysis of several studies revealed a high rate of compliance of the $K R A S$ gene mutational status in primary and metastatic tumors. In this paper we present a rare case of incompatibility of the KRAS mutations in the primary tumor located in the colon and metastatic changes in the liver.
\end{abstract}

Key words: advanced colorectal cancer, targeted therapy, KRAS gene.

\section{Introduction}

Colorectal cancer is one of the most common malignancies worldwide and is the second-leading cause of cancer morbidity among both men and women. In Poland more than 7000 men and 6000 women are diagnosed with colorectal cancer every year [1].

In the last decade the average survival of patients diagnosed with metastatic colorectal cancer increased from 12 to more than 20 months [2-4]. Most of the patients in this stage of the disease are eligible for systemic therapy. Thanks to the introduction of the multidrug regimens and targeted therapy (cetuximab, panitumumab, bewacizumab) into the clinical practice, 5year survival can be obtained in approximately $15 \%$ of patients [5-7]. The objective response to the first-line chemotherapy is achieved by 30 to $55 \%$ of the patients (Objective response rate, ORR), while only 5 to $25 \%$ of patients respond to the second line treatment [8]. The use of oxaliplatin or irinotecan based chemotherapy

Corresponding: Ewa Anna Kosakowska, Cancer

Center - Institute, Roentgena Str. 5, 02-781 Warsaw, Poland; tel./fax.: (+4822) 6440087, e-mail: ekosakowska@coi.waw.pl regimens as the second-line treatment prolongs overall survival time by 8 to 12 months $[9,10]$. Apart from the abdominal lymph nodes, liver is the most common site of the tumor spread. About $60 \%$ of the patients with colorectal cancer develop liver metastases and in approximately $25 \%$ of the patients liver is the only site of dissemination. The median 5-year survival rate following resection of liver metastases exceeds $30 \%$, suggesting that resectability of the metastases is an important prognostic factor [11]. In order to increase the number of patients undergoing resection of liver metastases, two or three drug chemotherapy regimens associated with targeted therapies are used in the preoperative and postoperative setting. The mutational analysis of the $K R A S$ gene is a necessary condition for the therapy based on the novel drugs like cetuximab or panitumumab, and the KRAS mutation predicts lack of response to these anti-EGFR antibodies [12-14]. The clinical benefit of bevacizumab added to chemotherapy in colorectal cancer is independent of the KRAS mutational status. This molecule may be used in the treatment of patients with a mutation in the KRAS gene (m KRAS) and without mutation (wt KRAS) as well [15]. In this paper we present a rare case of molecular 
differences, regarding KRAS gene mutational status, between a primary tumor and secondary lesions in a patient with metastatic colon cancer.

\section{A case report}

A 53-year old woman was admitted to a local GP unit because of abdominal pain. The ultrasound examination of the abdomen revealed the presence of metastatic lesions in both lobes of the liver, the biggest lesion was located in the segment VI/VII, measuring $58 \times 53 \mathrm{~mm}$. Computed tomography $(\mathrm{CT})$ confirmed the presence of liver metastases and also the signs of a neoplasmatic infiltration of the colon's splenic flexure. The patient underwent colonoscopy which revealed infiltration of the splenic flexure. Several bioptic samples were collected during endoscopic examination and the diagnosis of adenocarcinoma was confirmed histologically (adenocarcinoma G2). The left hemicolectomy connected with and resection of the pancreatic tail was performed. The histopathological report revealed: macroscopically a fragment of the colon measuring $25 \mathrm{~cm}$ with the ulcerated tumor and the enlarged lymph nodes in the peri-intestinal tissue, microscopically: moderately differentiated adenocarcinoma (adenocarcinoma mucinosum G-2), infiltrating the entire thickness of the intestinal wall with penetration into the peri-intestinal adipose tissue (T3) and obvious vascular invasion. The distal margins were free of the cancer infiltration. There were no metastases in the lymph nodes $(0 / 8$-N0) and the liver dissemination was histologically confirmed (metastases adenocarcinomatosae). Immunohistochemical analysis revealed EGFR (epidermal growth factor receptor) expression, with moderate and strong intensity of EGFR-membranous staining in $70 \%$ of the cancer cells (Fig. 2).

The KRAS gene mutational analysis, using the direct sequencing, revealed a mutation in codon 12 (Fig. 1). Final Diagnosis: pT3N0M1-HEP. The serum levels of tumor markers CEA and CA 19.9 were 3.3 $\mathrm{ng} / \mathrm{ml}$ and $1041.3 \mathrm{IU} / \mathrm{ml}$ respectively. Because of the difficulties in administering intravenous chemotherapy the patient received an oral chemotherapy capecitabine (dose $1250 \mathrm{mg} / \mathrm{m}^{2} \times$ daily for 14 days, the 21-day rhythm of treatment) in the Maria SklodowskaCurie Memorial Cancer Center, Warsaw. After administration of 2 cycles of the chemotherapy, a reduction of serum CA 19.9 to the level of $354.1 \mathrm{IU} / \mathrm{ml}$ was achieved, and abdominal ultrasonography revealed a partial regression of the metastatic lesions $(40 \%)$. Due to the observed toxicity - grade 2 palmar-plantar erythrodysesthesia (hand-foot syndrome), the dose of capecitabine was reduced to $2 \times 1000 \mathrm{mg} / \mathrm{m}^{2}$. After 5 cycles, there was a further reduction in serum CA 19.9 to the level of $106.1 \mathrm{IU} / \mathrm{ml}$. The computed tomography of the abdomen and pelvis, performed in December 2007 , demonstrated a regression of changes by $40 \%$. After another 3 cycles of chemotherapy, CT assessment revealed further regression of changes by $10 \%$, CA 19.9 serum concentration reached $122 \mathrm{IU} / \mathrm{ml}$ and CEA remained within the normal range $-2.5 \mathrm{ng} / \mathrm{ml}$. In March 2008, an unanatomical liver tumor resection on the border of segments IV and V was performed, and the metastases in segments II and VIII were thermoablated. During the surgery a lesion at the liver hilum, not described in the imaging studies, was found. Postoperatively, the patient received a second line palliative chemotherapy: FOLFOX plus bevacizumab (oxaliplatin $85 \mathrm{mg} / \mathrm{m}^{2}$, leucovorin $200 \mathrm{mg} / \mathrm{m}^{2}, 5$-fluorouracil $400 \mathrm{mg} / \mathrm{m}^{2}$ - a bolus, 5-flourouracyl 600 $\mathrm{mg} / \mathrm{m}^{2}$ - 48-hour infusion, bevacizumab $5 \mathrm{mg} / \mathrm{kg}$ a cycle, repeated every 14 days). The Ca 19.9 serum concentration before treatment was $457.3 \mathrm{IU} / \mathrm{ml}$. After 2 cycles the level decreased to $230 \mathrm{IU} / \mathrm{ml}$. A tolerance of treatment was good. The imaging studies performed after 5 cycles revealed a stabilization of the disease. During further treatment with oxaliplatin, the allergic reaction appeared (fever, chills and epigastric pain during the drug infusion), the drug dose was reduced and during the next cycles the dexamethasone pretreatment was administered. After administration of the next 5 cycles of chemotherapy, the imaging study revealed resectability of the other liver changes. After 14 cycles of chemotherapy FOLFOX + bevacizumab, a surgical treatment of liver metastatic lesions was carried out. A resection of a metastatic lesion located in the II and III liver segment, with an adequate margin was performed. The removed tumor adhered to the hepatic vein ( $\mathrm{R} 1$ resection). The postoperative pathological examination confirmed the diagnosis of metastatic adenocarcinoma of the colon. Two months after the surgery, the serum concentration of CA 19.9 was 6032 $\mathrm{IU} / \mathrm{ml}$. Re-examination of EGFR expression in the metastatic liver tumor was performed (tubular adenocarcinoma metastaticum), in which no presence of this receptor was found (negative reaction) (Fig. 2), and there were no mutations in codons 12 and 13 of the $K R A S$ gene (Fig. 1). Given the good response to the first line chemotherapy, an expected result of the bevacizumab treatment, no availability of anti-EGFR treatment, the patient received capecitabine in combination with bevacizumab. The abdominal ultrasound examination revealed a conglomerate of the lesions in the left liver lobe measuring $119 \times 70 \mathrm{~mm}$. After administration of 2 cycles of the treatment, a decrease in the serum level of CA 19.9 to $3025 \mathrm{IU} / \mathrm{ml}$ was obtained and a regression of the liver lesion by $25 \%$. Efficacy assessment of the chemotherapy was performed after 4 cycles, a general deterioration of clinical status was observed, the ultrasound examination of the liver revealed disease progression. The patient received 
Primary tumor

Metastatic site

G12D (35 G $\rightarrow$ T)
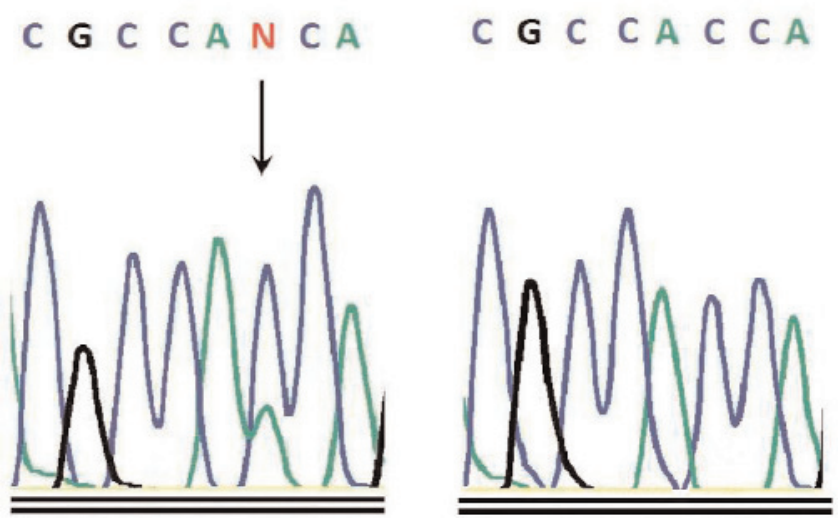

Fig. 1. Mutations of the KRAS gene. Identification of the somatic KRAS codon 12 mutant sequence $(\mathrm{G} \rightarrow \mathrm{T}$ at nucleotide 35$)$ in a primary tumor of colorectal cancer. Sequences are shown in the reverse direction and the arrow indicates the position of the mutation.

irinotecan monotherapy as the forth line treatment. 2 cycles were administered, without providing the therapeutic effect. The chemotherapy was terminated. Patient died in September 2009. Overall survival in the metastatic stage of disease was 27 months.

\section{Materials and methods}

Tumor specimens and histological examination. Tumor specimens were collected from colorectal cancer patient - primary tumor and related metastatic site in the liver. Tissues were fixed in buffered formalin for hematoxylin and eosin $(\mathrm{H}+\mathrm{E})$ staining. Serial $5 \mu \mathrm{m}$-thick sections of the fixed tissue were cut and stained with $\mathrm{H}+\mathrm{E}$. These specimens were used for routine histopathological examination. Sections of a paraffin block corresponding to one representative area of the tumor were stained with haematoxylin/eosin, and the presence of tumor tissue was verified by an experienced pathologist. Subsequently, tissue samples from at least three serial sections were macrodissected to ensure that specimens contained at least $80 \%$ tumor cells.

DNA isolation. DNA from paraffin-embedded colorectal tumor specimens was prepared from 10-30 $\mu \mathrm{m}$ sections after macrodissection, resulting in selection of at least $80 \%$ tumor cells. Tumor samples were extracted with xylene and ethanol to remove paraffin and placed in $1 \% \mathrm{SDS} /$ proteinase $\mathrm{K}(10 \mathrm{mg} / \mathrm{ml})$ at $56^{\circ} \mathrm{C}$ overnight. DNA was iso- lated using the The NucliSens easyMAG platform (bioMérieux) for automated nucleic acid extraction.

Analysis of K-RAS mutation. Mutation analysis of $K R A S$ codons 12 and 13 was carried out by direct sequencing of amplified PCRproducts spanning respective codons. DNA was amplified for KRAS exon 1 using specific primers: forward FS 5'- TCA TTA TTT TTA TTA TAA GGC CTG CTG - 3', reverse RS 5'-CAA GAT TTA CCT CTA TTG TTG GAT CA-3'. Polymerase chain reaction (PCR) was carried out in a total volume of $10 \mu \mathrm{l}$ contained $2 \mu \mathrm{l}$ of the extracted genomic DNA using $1 \mathrm{U}$ of Taq DNA polymerase (EURx Ltd., Gdansk, Poland). The initial denaturing step was at $95^{\circ} \mathrm{C}$ for $10 \mathrm{~min}$, followed by 36 cycles of denaturing step at $95^{\circ} \mathrm{C}$ for $30 \mathrm{~s}$, annealing step at $56^{\circ} \mathrm{C}$ for $30 \mathrm{~s}$ and extension step at $72^{\circ} \mathrm{C}$ for $30 \mathrm{~s}$, ending with a final extension step at $72^{\circ} \mathrm{C}$ for $5 \mathrm{~min}$. Amplification products were purified using the

DNA Gel-Out Kit (DNA GDANSK, Gdynia, Poland). Automated sequencing was carried out using the Big Dye Terminator Cycle Sequencing kit version 3.1 (Applied Biosystems, Warsaw, Poland). Sequencing reactions were purified using the ExTerminator Kit (DNA GDANSK, Gdynia, Poland), and analysed on an ABI PRISM 377 DNA sequencer (Applied Biosystems, Warsaw, Poland). A wild-type control DNA sample (without KRAS codon 12 mutation) and a known mutation sample were included in the experiment. The mutation was confirmed by sequencing at least two independent PCR products.

Immunohistochemistry for EGFR. The paraffin-embedded tissues were cut at $5 \mu \mathrm{m}$ thickness. Immunohistochemical staining of sections was performed using the immunohistochemical system kit EGFR pharmDx (Dako Poland), which included proteinase K, peroxidase block, EGFR pharmDx monoclonal mouse antibody (clone 2-18C9), mouse IgG1 negative control reagent, labeled polymer $\mathrm{HRP}, \mathrm{DAB}+$ substrate buffer, liquid $\mathrm{DAB}+$ chromogen, DakoCytomation wash solution $10 \times$, and EGFR pharmDx control slides. The immunostaining was performed according to instructions supplied by the manufacturer. The controls used for the validation of EGFR assay were included in EGFR pharmDx: negative control reagent, positive and negative control cell preparation. EGFR expression was detected as membranous and/or cytoplasmic brown staining of neoplastic cells with various intensity. Positivity for EGFR expression was defined as any membrane staining above background level. Both the primary and metastatic neoplasm were considered positive when $>1 \%$ of the tumor cells had membranous staining. A specific membrane staining in less than $1 \%$ of neoplastic cells was defined as EGFR-negative.

Ethical issues. The $K R A S$ gene mutational status analysis is a procedure performed routinely and does not require a bioethics committee approval.
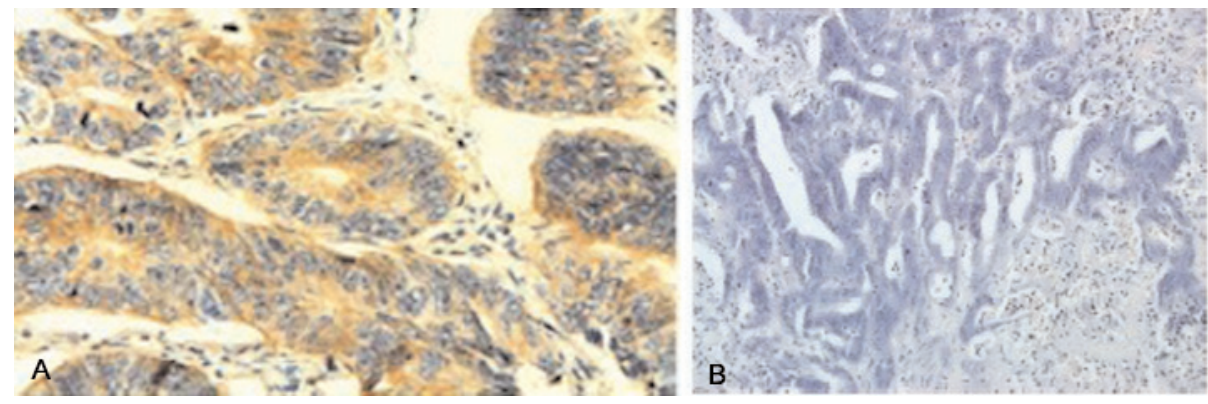

Fig. 2. Epidermal growth factor receptor (EGFR) staining by immunohistochemistry of primary and secondary colon adenocarcinoma. (A) EGFR expression - primary colon adenocarcinoma; (B) EGFR negative - secondary colon adenocarcinoma to the liver, $(\mathbf{C})$ control of staining - the immunohistochemical system kit EGFR pharmDx of the liver cells. 


\section{Discussion}

Mutations in the RAS genes family (HRAS, NRAS and KRAS) are the most common oncogenic changes, that are present in a majority of the human cancers [16]. Point mutations in the critical coding sequences of the KRAS gene, identified mainly in codon 12 and $13[17,18]$, are observed in approximately $30-45 \%$ cases of primary colorectal cancer $[19,20]$. Constitutive activation of the RAS protein, as a result of mutations in the KRAS gene, leads to stimulation of the signaling pathways (PI3K/PTEN/AKT and RAF/MEK/ ERK) responsible for cell growth, inhibition of apoptosis, differentiation, proliferation of cancer cells, invasion and metastases [21,22]. Signaling pathways, activated via the hyperactive RAS protein, become independent and cannot be controlled at the level of EGFR [23]. Mutational status of the KRAS gene is a key predictive factor for response to the monoclonal antibodies against EGFR [24-26]. Targeted therapy, based on a direct blocking of the epidermal growth factor receptor (EGFR) using specific monoclonal antibodies (cetuximab and panitumumab), leads to the inhibition of EGFR signaling cascade [27-29]. Results of the independent clinical trials led to the EMEA (European Medicines Agency) approval for the use of cetuximab and panitumumab in the treatment of patients with metastatic colon cancer $[25,29,30]$. According to the approval, therapy with the antiEGFR antibodies is confined only to the patients with a positive immunohistochemical EGFR reaction, when the presence of the KRAS gene mutations is excluded $[31,32]$. Subsequently, the retrospective analyzes have demonstrated the lack of correlation between EGFR expression status, assessed by immunohistochemical staining, and response to the therapy $[23,30,33]$. Recent studies, evaluating the clinical response in patients with advanced or metastatic colorectal cancer, showed that the presence of KRAS mutation is associated with the lack of response to cetuximab [24,33-37]. Patients without mutations in the KRAS gene gain therapeutic benefit from the use of cetuximab [38]. Similarly, the use of panitumumab in patients with the presence of $K R A S$ mutations, did not bring the expected therapeutic benefit [24].

Patients with the tumors not presenting mutation in the $K R A S$ gene (KRAS wild type - WT KRAS), show a good response to the targeted therapy, effecting in an increase of progression free survival (PFS) and overall survival (OS) $[24,30]$. However, a significant proportion of patients with the wild-type $K R A S$ (KRAS-WT) shows little or no response to the anti-EGFR treatment [39]. Clinical-molecular data clearly indicate the existence of other mechanisms of cancer cells' resistance to the therapy with anti-EGFR monoclonal antibodies. Molecular evaluation of the KRAS status in the metastatic site, instead of the primary tumor, may have a great clinical significance. The presence of the KRAS oncogene mutations in the metastatic tumor, in the absence of mutations in the primary lesion, could at least partly explain the resistance of cancer cells to the treatment.

Until now, few studies comparing the biological characteristics, in terms of EGFR, AKT and MAPK protein expression [40], of primary tumors and corresponding metastases, have been conducted $[41,42]$. Immunohistochemical findings indicate a considerable diversity of biological processes involved in the neoplastic transformation of the primary and metastatic tumors. Data presented in our case report confirm the differences in the EGFR expression between the primary and secondary tumors. Immunohistochemical studies showed a positive EGFR reaction in the primary tumor and a lack of EGFR expression in the metastatic site. Incompatibility is likely due to the significant heterogeneity of the tumors in terms of the EGFR expression. Previous studies assessing the mutational status of the KRAS gene in the colorectal cancer, were confined almost exclusively to the analysis of the primary tumors. The few reports assessing a degree of compliance of the KRAS mutational status in the primary and secondary lesions, are insufficient and vary significantly. Artale et al described a high compliance of mutations in the KRAS and BRAF genes in the primary site and distant metastases of colorectal cancer (92\%) [41].The study population comprised of only 48 patients and the analysis was confined mostly to the distant lesions. Santini and colleagues analyzed the degree of conformity of KRAS mutations in primary tumors and distant metastases in the group of 99 patients, confirming earlier reports of Artale et al [42]. The results of the analysis showed a high rate of compliance of the KRAS mutational status (96\%). Only in four cases, of which three were related to hepatic metastases, there was a lack of compliance. Similar studies conducted by Molinari et al, also revealed a high-factor of compliance of the KRAS mutational model [43], although the study included a small group of patients. Our case report presents a mismatch of the $K R A S$ mutational status between the primary tumor and the metastatic site in the liver. Molecular analysis revealed the presence of mutations in codon 12 of the $K R A S$ gene (G12D) in the primary tumor and a lack of mutationsin the metastatic lesion. Velho et al conducted an analysis of the incidence of KRAS and BRAF mutations in a primary site and corresponding metastases to the lymph nodes in the group of 250 patients with sporadic colorectal cancer [44]. The obtained results differed significantly from the results presented by Artale et al. The frequency of the KRAS mutations in the lymph nodes metastases was significantly higher $(82.1 \%)$ comparing to the primary tumors $(55 \%)$. Molecular data provided by Velho et al support the 
hypothesis that cancer cells acquire $K R A S$ mutations in the early stages of the cancer progression [44]. The primary tumor is likely to consist of different populations of tumor cells in terms of the $K R A S$ mutational status. Tumor cells presenting the KRAS mutations (KRAS-M) show a higher rate of proliferation, differentiation and resistance to apoptosis [22]. Only a small percentage of tumor cells is able to survive a migration and subsequently to initiate the development of the secondary lesions. The M-KRAS cancer cells show a better adaptation to the adverse conditions of a migration and colonization of the nearby lymph nodes and the distant tissues. A mechanism of the recruitment and clonal selection of the tumor cells, in terms of the KRAS mutational status, may be an important process, explaining a value of the KRAS mutation in the metastatic cascade [45]. The results of these studies may have a great clinical significance, presenting one of the possible mechanisms of resistance to anti-EGFR therapy of the patients with metastatic colon cancer and WT-KRAS. The presence of KRAS mutations in the metastatic site may be an important factor, leading to a resistance to the treatment. A lack of $K R A S$ mutations in the secondary tumors, despite the presence of a mutation in the primary lesion, may become an additional predictive factor, allowing better selection of patients undergoing the anti-EGFR therapy. Additional studies, evaluating the mutational status of the KRAS gene in the secondary lesions may be helpful in selection of the patients eligible for this type of anti-cancer therapy.

\section{References}

[ 1] Wojciechowska U, Didkowska J, Tarkowski W. Nowotwory złośliwe w Polsce 2004 roku. Warsaw: Centrum Onkologii Instytut; 2006.

[2] Cunningam D, Pyrhonen S, James RD et al. Randomised trial of irinotecan plus supportive care versus supportive care alone after flurouracil failure for patients with metastatic colorectal cancer. Lancet. 1998;352:1407-1412.

[3] Hurvitz H, Fehrenbacher L, Novoty W et al. Addition of bevacizumab (rhu MAb VEGF) to bolus IFL in the first-line treatment of patients with metastatic colorectal cancer: results of a randomized phase III trial. New Engl J Med. 2004;350:2335-2342.

[4] Nordic Gastrointestinal Tumor Adjuvant Therapy Group. Expectancy or primary chemotherapy in patients with advanced asymptomatic colorectal cancer: randomized trial. $J$ Clin Oncol. 1992;10:904-911.

[5] Ciardiello F, De Vita F, Orditura M et al. Cetuximab in the treatment of colorectal cancer colorectal cancer. Future Oncol. 2005;1:173-81.

[ 6] Van Cutsem E, Peeters M, Siena S. Open-label phase III trial of panitumumab plus best supportive care compared with best supportive care alone in patients with chemotherapyrefractory metastatic colorectal cancer. J Clin Oncol. 2007;25:1658-64.

[ 7] Saltz LB, Clarke S, Diaz-Dubio E et al. Bevacizumab in combination with oxaliplatin-base chemotherapy in metastatic colorectal cancer randomized phase III study. J Clin Oncol. 2008;26:2013-2019.
[ 8] Biasco G, Derenzini E, Grazi GL et al. Treatment of hepatic metastases from colorectal cancer: Many doubts, some certainties. Cancer Treat Rev. 2006;32:214-228.

[9] Maindrault-Goebel et al. Oxaliplatin addend to simplified bimonthly leucovorin and 5-fluorouracil regiment as secondline therapy for metastatic colorectal cancer (FOLFOX6). GERCOR. Eur J Cancer. 1999;35:1338-1342.

[10] Tournigand C, Andre T, Achille E et al. FOLFIRI followed by FOLFOX6 or the reverse sequence in advanced colorectal cancer: a randomized GERCOR study. J Clin Oncol. 2004;22:229-237.

[11] Figueras J, Valls C, Rafecas A et al. Resection rate and effect of postoperative chemotherapy on survival after surgery for colorectal liver metastases. Br J Surg. 2001;88:980-985.

[12] Baselga J. The EGFR as a target for anticancer therapy focus on cetuximab. Eur J Cancer. 2001;37:Suppl 4:S16-S22.

[13] Jonker DJ, O'Callaghan CJ, Karapetis CS et al. Cetuximab for the treatment of colorectal cancer. New Engl J Med. 2007;357:2040-8.

[14] Bokemeyer C, Bondarenko I, Hartmann JT et al. KRAS status and efficacy of first-line treatment of patients with metastatic colorectal cancer (mCRC) with FOLFOX with or without cetuximab: The OPUS experience. J Clin Oncol. 2008;26:178s, (suppl, abstr 4000).

[15] Hurwitz HI. The clinical benefit of bevacizumab in metastatic colorectal cancer is independent of KRAS mutation status: analysis of phase III study of bevacizumab with chemotherapy in previously untreated metastatic colorectal cancer. Oncologist. 2009;1:22-28.

[16] Dobrzycka B, Terlikowski S, Kowalczuk O et al. Mutations in the KRAS gene in ovarian tumors. Folia Histochem Cytobiol. 2009;47(2):221-224.

[17] Wu CM, Tang R, Wang JY, Changchien CR et al. Frequency and spectrum of K-RAS codons 12 and 13 mutations in colorectal adenocarcinomas from Taiwan. Cancer Genet Cytogenet. 2005;158(1):55-60.

[18] Kraus MC, Seelig MH, Linnemann U et al. The balanced induction of K-ras codon 12 and 13 mutations in mucosa differs from their ratio in neoplastic tissues. Int $J$ Oncol. 2006;29(4):957-64.

[19] Brink M, de Goeij AF, Weijenberg MP et al. K-ras oncogene mutations in sporadic colorectal cancer in The Netherlands Cohort Study. Carcinogenesis. 2003;24:703-710.

[20] Andreyev HJ, Norman AR, Cunningham D et al. Kirsten ras mutations in patients with colorectal cancer: The 'RASCAL II' study. Br J Cancer. 2001;85:692-696.

[21] Lewis TS, Shapiro PS, Ahn NG. Signal transduction through MAP kinase cascades. Adv Cancer Res. 1998;74:49-139.

[22] Benvenuti S, Sartore-Bianchi A, Di Nicolantonio F et al. Oncogenic activation of the RAS/RAF signaling pathway impairs the response of metastatic colorectal cancers to antiepidermal growth factor receptor antibody therapies. Cancer Res. 2007;67:2643-2648.

[23] Scartozzi M, Bearzi I, Berardi R et al. Epidermal growth factor receptor (EGFR) status in primary colorectal tumors does not correlate with EGFR expression in related metastatic sites: Implications for treatment with EGFR-targeted monoclonal antibodies. J Clin Oncol. 2004;22:47724778.

[24] Amado RG, Wolf M, Peeters M et al. Wild-type KRAS is required for panitumumab efficacy in patients with metastatic colorectal cancer. J Clin Oncol. 2008;26:1626-34.

[25] Di Fiore F, Blanchard F, Charbonnier F et al. Clinical relevance of KRAS mutation detection in metastatic colorectal cancer treated by cetuximab plus chemotherapy. Br J Cancer. 2007;96:1166-9.

[26] Lievre A, Bachet JB, Boige V et al. KRAS mutations as an independent prognostic factor in patients with advanced col- 
orectal cancer treated with cetuximab. J Clin Oncol. 2008;26:374-379.

[27] Baselga J. The EGFR as a target for anticancer therapy focus on cetuximab. Eur J Cancer. 2001;37:Suppl 4:S16-S22.

[28] Khambata-Ford S, Garrett CR, Meropol NJ et al. Expression of epiregulin and amphiregulin and K-ras mutation status predict disease control in metastatic colorectal cancer patients treated with cetuximab. J Clin Oncol. 2007;25:3230-3237.

[29] Cunningham D, Humblet Y, Siena S et al. Cetuximab monotherapy and cetuximab plus irinotecan in irinotecanrefractory metastatic colorectal cancer. New Engl J Med. 2004;351:337-345.

[30] European Medicines Agency: Questions and answers on the marketing authorization for Vectibix. Available at: http:/www.emea.europa.eu/pdfs/human/opinion/40511307en .pdf, Accessed September 20, 2007.

[31] European Medicines Agency: Committee for Medicinal Products for Human Use post-authorisation summary of positive opinion for Erbitux. Available at: http://www.emea.europa.eu/ /pdfs/human/opinion/Erbitux_28040208en.pdf, Accessed May 30, 2008.

[32] Van Cutsem E, Lang I, D'haens G et al. KRAS status and efficacy in the first-line treatment of patients with metastatic colorectal cancer (mCRC) treated with FOLFIRI with or without cetuximab: The CRYSTAL experience. $J$ Clin Oncol. 2008;26:5s, (suppl, abstr 2).

[33] Oudejans JJ, Slebos RJ, Zoetmulder FA et al. Differential activation of ras genes by point mutation in human colon cancer with metastases to either lung or liver. Int $J$ Cancer. 1991;49:875-879.

[34] Suchy B, Zietz C, Rabes HM. K-ras point mutations in human colorectal carcinomas: Relation to aneuploidy and metastasis. Int J Cancer. 1992;52:30-33.

[35] Al-Mulla F, Going JJ, Sowden ET et al. Heterogeneity of mutant versus wild-type Ki-ras in primary and metastatic colorectal carcinomas, and association of codon-12 valine with early mortality. J Pathol. 1998;185:130-138.

[36] Karapetis CS, Khambata-Ford S, Jonker DJ et al. K-ras mutations and benefit from cetuximab in advanced colorectal cancer. New Engl J Med. 2008; 359(17):1757-65.
[37] de Reynie`s A, Boige V, Milano G et al. KRAS mutation signature in colorectal tumors significantly overlaps with the cetuximab response signature. J Clin Oncol. 2008;26:22282230 .

[38] Scartozzi M, Bearzi I, Berardi R et al. Epidermal growth factor receptor (EGFR) downstream signalling pathway in primary colorectal tumours and related metastatic sites: Optimising EGFR-targeted treatment options. Br J Cancer. 2007; 97:92-97.

[39] Scartozzi M, Bearzi I, Berardi R et al. Epidermal growth factor receptor (EGFR) status in primary colorectal tumors does not correlate with EGFR expression in related metastatic sites: Implications for treatment with EGFR-targeted monoclonal antibodies. J Clin Oncol. 2004; 22:4772-4778.

[40] Cappuzzo F, Finocchiaro G, Rossi E et al. EGFR FISH assay predicts for response to cetuximab in chemotherapy refractory colorectal cancer patients. Ann Oncol. 2008; 19:717-723.

[41] Artale S, Sartore-Bianchi A, Veronese SM et al. Mutations of KRAS and BRAF in primary and matched metastatic sites of colorectal cancer. J Clin Oncol. 2008:1;26(25):4217-9.

[42] Santini D, Loupakis F, Vincenzi B et al. High concordance of KRAS status between primary colorectal tumors and related metastatic sites: implications for clinical practice. Oncologist. 2008;13(12):1270-5. Epub 2008 Dec 4.

[43] Molinari F, Martin V, Bordoni A et al. Analysis of epidermal growth factor receptor (EGFR) gene status and protein expression, and K-ras gene mutations in metastatic colorectal cancer patients: Comparison between primary tumor and related metastatic sites. Ann Oncol. 2008;19(suppl. 1):i10i25.

[44] Vehlo S, Oliviera C, Seruca R. KRAS mutations and anti-epidermal growth factor receptor therapy in colorectal cancer with lymph node metastases. J Clin Oncol. 2009;1:158-9.

[45] Oliveira C, Velho S, Moutinho C et al. KRAS and BRAF oncogenic mutations in MSS colorectal carcinoma progression. Oncogene. 2007;26:158-163.

Submitted: 14 May, 2010 Accepted after reviews: 7 September, 2010 